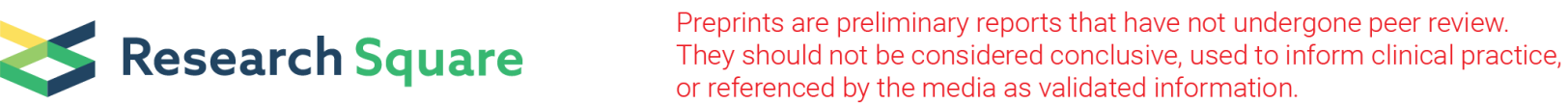

\section{Fabrication of Reduced Graphene Oxide-Cellulose Nanofibers Based Hybrid Film with Good Hydrophilicity and Conductivity as Electrodes of Supercapacitor}

\section{chuanyin xiong ( $\nabla$ xiongchuanyin@126.com )}

Shaanxi University of Science and Technology

\section{Congmin Zheng}

Shaanxi University of Science and Technology Xi\'an Campus: Shaanxi University of Science and Technology

Shuangxi Nie

Guangxi University

Chengrong Qin

Guangxi University

Lei Dai

Shaanxi University of Science and Technology Xi\'an Campus: Shaanxi University of Science and Technology

\section{Yongjian Xu}

Shaanxi University of Science and Technology Xi\'an Campus: Shaanxi University of Science and Technology

\section{Yonghao Ni}

University of New Brunswick

\section{Original Research}

Keywords: Cellulose nanofibers, reduced graphene oxide, Film, Chemical reduction, Supercapacitor, Flexibility

Posted Date: February 3rd, 2021

DOl: https://doi.org/10.21203/rs.3.rs-188518/v1

License: (c) (i) This work is licensed under a Creative Commons Attribution 4.0 International License. Read Full License 
Version of Record: A version of this preprint was published at Cellulose on March 2nd, 2021. See the published version at https://doi.org/10.1007/s10570-021-03791-0. 


\section{Abstract}

In this research, a kind of non-carbonized reduced graphene oxide (RGO)-cellulose nanofibers (CNF) film is constructed by a combination of filtration and chemical reduction. In the hybrid, RGO enhances conductivity of CNF, and CNF as an idea spacer not only prevents stacking of RGO but also gives the film good mechanical properties including mechanical strength and flexibility. The synergistic effect of both endows the film with good electrochemical storage performance and outstanding mechanical properties. The hybrid film can be directly assembled into a symmetrical supercapacitor that shows an outstanding cycle stability, excellent rate performance, good mechanical strength and flexibility. Moreover, the film presents a high specific capacitance of $120 \mathrm{mF} \mathrm{cm}{ }^{-2}$, energy density of $536 \mu \mathrm{Wh} \mathrm{cm}^{-2}\left(32 \mathrm{Wh} \mathrm{kg}^{-1}\right)$ and power density of $193 \mathrm{~mW} \mathrm{~cm}^{-2}\left(53 \mathrm{~kW} \mathrm{~kg}^{-1}\right)$. Additionally, the film can be used as a substrate to graft other components.

\section{Introduction}

As a new type of energy storage device between traditional capacitor and battery, supercapacitor has the advantages of fast charge discharge rate, long cycle life, high power density (to meet the requirements of high current and high power operation) and so on. It is considered to be a promising energy source (Dang et al. 2018; Peng et al. 2020; Xiong et al. 2020; Wu et al. 2020; Xiong et al. 2019; Liu et al. 2020). Especially in recent years, with the rapid development of flexible wearable electronic devices, various kinds of flexible wearable electronic devices have emerged one after another. As a new portable energy storage device, the research and application of supercapacitors have attracted much attention (Xiong et al. 2020; Sun et al. 2020; Xiong et al. 2020; Zhang et al. 2020; Xiong et al. 2020; Zhang et al. 2020; Xiong et al. 2020).

As one of the most important core components of supercapacitor, the performance of electrode material has a crucial impact on the electrochemical storage performance of the whole supercapacitor (Nie et al. 2020; Li et al. 2020; Chen et al. 2020; Xiao et al. 2020; Bi et al. 2020). To the best of knowledge, the electrode materials of supercapacitors can classified into into two types according to their charge storage mechanism, namely, electric double layer and pseudo capacitor electrode materials (Thi Suong et al. 2020; Xiong et al. 2016; Zhu et al. 2020). The former mainly includes activated carbon (Wei et al. 2020), graphene (GR) (Cheng et al. 2020), carbon nanotube (CNT) (Fan et al. 2020), etc. The latter mainly includes metal oxides (manganese, nickel, cobalt and other oxides) (Xiong et al. 2020; Li et al. 2019; Wang et al. 2019), conductive polymers (polyaniline (PANI) and polypyrrole (PPy) (Du et al. 2020; Tong et al. 2019) and other pseudo capacitance materials (multilayer metal hydroxide, metal organic framework, two-dimensional transition metal carbides or carbonitrides, etc.) (Xiao et al. 2020; Ten Elshof et al. 2016; Wu et al. 2020; Deysher et al. 2020). Thereinto, GR, as a two-dimensional single-layer carbon atom crystal, has been characterized by its good conductivity, large specific surface area (theoretical value can reach $2630 \mathrm{~m}^{2} \mathrm{~g}^{-1}$ ), high mass and area specific capacity (theoretical value can reach $500 \mathrm{~F} \mathrm{~g}^{-1}$ and $21 \mu \mathrm{F}$ $\mathrm{cm}^{-2}$ ), high mass and volume energy density (theoretical value can reach $169 \mathrm{wh} \mathrm{kg}^{-1}$ and $303 \mathrm{wh} \mathrm{L}^{-1}$ 
respectively)) (El-Kady et al. 2016), reaching the upper limit of all carbon based electric double layer capacitors, which is higher than other carbon materials, and is an ideal material for manufacturing supercapacitors. However, due to the strong van der Waals force and $\pi-\pi$ bond between GR layers, GR tends to agglomerate, significantly reducing its specific surface area (generally only $500 \mathrm{~m}^{2} \mathrm{~g}^{-1}$, or even lower), which affects its electrochemical energy storage performance as a supercapacitor electrode: the specific capacity is only $100 \sim 200 \mathrm{~F} \mathrm{~g}^{-1}$, the mass energy density is only $15 \sim 35 \mathrm{wh} \mathrm{kg}^{-1}$, volumetric energy density $<60 \mathrm{Wh} \mathrm{L}^{-1}$ [El-Kady et al. 2016; Zhao et al. 2017]. These indicators show that the electrochemical energy storage performance of GR supercapacitor is far lower than its theoretical value, and there is still a lot of room for improvement. In order to solve the problem of low performance of GR supercapacitors, it is one of the common strategies to construct 3D GR-spacer composite electrode materials by introducing spacers between GR layers, mainly including carbon materials (Xiong et al. 2016), metal oxides (Xiong et al. 2019) and conductive polymers (Xiong et al. 2020). Indeed, the introduction of these spacers improves the utilization efficiency of graphene to some extent, and the GRbased composites with good electrochemical storage properties are obtained. However, from the perspective of practical application, for most of 3D GR based composite films constructed by conventional electric double layer and pseudocapacitor as spacers, there are still many aspects to be further improved, including film-forming ability, mechanical properties, electrochemical stability, flexibility and so on. Therefore, it is very important to select the appropriate spacers for the construction of 3D GR based composites with good comprehensive properties, so as to better meet the practical application. As a kind of sustainable biomass resource with extensive sources, cellulose nanofibers (CNF) based materials have been widely studied and applied by many researchers due to its advantages of light weight, regeneration, good dispersion, good film-forming ability and excellent mechanical properties. It has the potential to be a good spacer to combined with GR.

In this study, a kind of non-carbonized reduced graphene oxide (RGO)-CNF hybrid film is fabricated by a combination of filtration and chemical reduction. In the hybrid, introduction of CNF not only prevents the stacking of RGO but also endows the composite film good mechanical properties including mechanical strength and flexibility. Moreover, the cross-linked CNF network structure can be also provide a lot of space for the storage and transmission of electrolyte ions. In addition, RGO enhances the conductivity of CNF. Consequently, The as-fabricated hybrid film directly assembled into a symmetrical supercapacitor displays a good cycle stability, outstanding rate performance, good mechanical strength and flexibility. Specifically, the hybrid film shows a high specific capacitance of $120 \mathrm{mF} \mathrm{cm}^{-2}\left(242 \mathrm{~F} \mathrm{~g}^{-1}\right)$, energy efficiency of $80 \%$, energy density of $536 \mu \mathrm{Wh} \mathrm{cm}^{-2}\left(32 \mathrm{Wh} \mathrm{kg}^{-1}\right)$ and power density of $193 \mathrm{~mW} \mathrm{~cm}^{-2}(53$ $\mathrm{kW} \mathrm{kg}^{-1}$ ). More importantly, the hybrid film can be served as a substrate to load other components. Furthermore, RGO-CNF@PANI hybrid film is fabricated by a simple electropolymerization method. The composite not only perfectly inherits the advantages of the RGO-CNF hybrid, but also shows a greater improvement in energy storage performance (energy and power density of $825 \mu \mathrm{Wh} \mathrm{cm}{ }^{-2}$ and $338 \mathrm{~mW}$ $\left.\mathrm{cm}^{-2}\right)$. 


\section{Experiment}

\subsection{Preparation of Graphene oxide (GO)-CNF film}

GO was prepared by Hummers method (Marcano et al. 2010). CNF solution of $0.1 \mathrm{wt} \%$ was purchased from Qihong Technology Co., Ltd in china. Different contents of CNF (30, 50, 70 \%) were added into GO solution, and then different GO-CNF films were fabricated by ultrasonic and suction filtration. The asobtained GO-CNF films were labeled as GO-CNF30, GO-CNF50 and GO-CNF70, respectively. As a contrast, pure GO film was also prepared according to the above method.

\subsection{Fabrication of RGO-CNF film}

The prepared GO-CNF film samples were further reduced to RGO-CNF by dropping hydrogen iodide solution. The reduced RGO-CNF film were denoted as RGO-CNF30, RGO-CNF50 and RGO-CNF70, respectively. The schematic diagram of the whole preparation process is shown in Fig. 1.

\subsection{Fabrication of RGO-CNF@PANI film}

The prepared RGO-CNF film samples were used as substrates to load PANI by electropolymerization according to the same procedure in our previous work (Xiong et al. 2020).

\subsection{Self-assembled RGO-CNF (GO, GO-CNF) supercapacitors}

Two RGO-CNF (GO, GO-CNF) films were used as the positive and negative electrodes of supercapacitor, and separated by diaphragm. A simple RGO-CNF supercapacitor was successfully fabricated as shown in Fig. 2.

\subsection{Electrochemical measurement}

The electrochemical properties of the film samples prepared above were conducted on a $\mathrm{CHI} 760 \mathrm{E}$ electrochemical workstation (Shanghai, Chenhua). The $\mathrm{Li}_{2} \mathrm{SO}_{4}$ and $\mathrm{PVA} / \mathrm{H}_{3} \mathrm{PO}_{4}$ solution were selected as aqueous and solid electrolytes, respectively. The basic Cyclic voltammograms (CV), galvanostatic chargedischarge (GCD) and Electrochemical Impedance Spectroscopy (EIS) measurements were carried out according the same method in our previous work (Xiong et al. 2020). In addition, specific capacitance $C_{m}$ $\left(\mathrm{mF} \mathrm{cm}{ }^{-2}, \mathrm{~F} \mathrm{~g}^{-1}\right)$, energy density $\mathrm{W}\left(\mu \mathrm{Wh} \mathrm{cm}^{-2}, \mathrm{Wh} \mathrm{kg}^{-1}\right)$ and power density $\mathrm{P}\left(193 \mathrm{~mW} \mathrm{~cm}{ }^{-2}, \mathrm{~kW} \mathrm{~kg}^{-1}\right)$ were calculated from $\mathrm{CV}$ curves according to the equations displayed in our previous work (Xiong et al. 2016).

\section{Results And Discussion}

3.1. Characterization of morphology and components 
As a comparison, the pure CNF film, GO-CNF film and RGO-CNF film are prepared, and the corresponding digital graphs of these samples are presented in Fig. 3(a). It can be seen that the pure CNF displays white color with a little wrinkle, and after introduction of GO or RGO, a flat black pie is successfully prepared indicating that the addition of GO or RGO is beneficial to the formation of CNF film. Furthermore, the morphology of these samples are observed through SEM (Fig. 3(b-d)) and TEM (Fig. 3(e-g)). Apparently, the SEM image of pure CNF shows small wrinkles (Fig. 3(d)) due to the cross-linked network of CNF. The cross-linked network of CNF can be observed through TEM, which also demonstrates that the wrinkled CNF can be served as an ideal spacer to better block the stacking of GO. It can be seen clearly that after introduction of CNF the GO-CNF and RGO-CNF films both display obvious wrinkles as shown in Fig. 3(c, d), which further supports the above conclusions. Moreover, it can be seen from the TEM images (Fig. 3(f, g)) that the crossed-linked CNF can form some new pores between the GO layers, which is beneficial for the storage and transmission of electrolyte ions. In addition, it is noted that the obtained RGO-CNF film can be used as an ideal flexible substrate to load other components, such as metal oxide, conductive polymer. Here, conducting polyaniline (PANI) due to its large pseudocapacitance is regarded as a good candidate to graft onto the RGO-CNF film by electropolymerization. SEM images of the morphology of RGO-CNF film are shown in the support information Fig. S1(a-c). Apparently, there are a lot of PANI nanofibers on the surface of RGO-CNF film after electropolymerization. Moreover, these PANI nanofibers interweave with each other to form a porous network, which is conducive to the transport of electrolyte ions.

Furthermore, to check whether the RGO-CNF hybrid film is successfully prepared, it is very necessary to investigate the XRD and Raman spectrum. As a comparison, the related spectrum of GO-CNF film is also tested. Fig. 4(a, b) show the XRD and Raman test results of these samples, respectively. Obviously, in the XRD spectrum (Fig.4 (a)) there is an obvious characteristic peak at around $26^{\circ}$ as expected representing the diffraction of GO (Xiong et al. 2016). When GO is reduced to RGO, the intensity of characteristic peak of GO becomes weaker, and a new peak appears at about $10^{\circ} \mathrm{Q}$ which is the characteristic peak of RGO (Xiong et al. 2016). In addition, the Raman spectrum is displayed in Fig. 4(b), where the D, G and 2D peaks representing the characteristic peaks of GO and RGO (Xiong et al. 2016) appear respectively. However, after $\mathrm{GO}$ is reduced, the $\mathrm{I}_{D} / \mathrm{I}_{\mathrm{G}}$ value representing the defect degree of the material decreased, which also indicates that GO is effectively reduced. In addition, for the RGO-CNF-PANI hybrid, there are two peaks appeared in the low and high wavenumber regions of the raman spectra as shown in the support information Fig. S1(d), respectively. The peaks at 820 and $1480 \mathrm{~cm}^{-1}$ correspond to amine/imine deformation (C-N-C bending) and C-N stretching vibration, respectively (Xiong et al. 2019). These results demonstrate that the PANI has been successfully electropolymerized onto the RGO-CNF film.

\subsection{Physical properties test}

To the best of our knowledge, the hydrophilicity and conductivity of materials have important influence on their electrochemical storage performance. For comparison, the hydrophilicity of pure CNF, GO and RGO-CNF films are both measured as displayed in Fig. 5. Apparently, pure GO film exhibits the largest average contact angle, reflecting its good hydrophilicity. CNF film has poor hydrophilicity, and the 
hydrophilicity of the RGO-CNF film is greatly improved after adding GO. The improvement of hydrophilicity of RGO-CNF film is conducive to its better contact with electrolyte ions. In addition, to check the conductivity of RGO-CNF film, Fig. 6(a, b) display the resistance of GO-CNF and RGO-CNF films through multimeter test. Apparently, compared with the resistance value of GO-CNF film $55 \mathrm{k} \Omega$, the resistance value of RGO-CNF film is greatly reduced to only $0.51 \mathrm{k} \Omega$ (Fig. $6(\mathrm{a}, \mathrm{b})$ ), which proves that RGOCNF film has good conductivity. Fig. 6(c-e) display the bending of RGO-CNF films under different conditions. Obviously, RGO-CNF films exhibit good flexibility and even fold in half. Furthermore, the tensile properties of RGO and RGO-CNF films are compared as shown in Fig. 7, where it can be seen that the RGO-CNF film presents a big improvement in tensile strength from less than $1 \mathrm{Mpa}$ for pure RGO film to about $8 \mathrm{Mpa}$ for RGO-CNF film, demonstrating that the addition of CNF greatly improves the mechanical properties of composite films. In a word, by testing and analyzing the hydrophilicity, electrical conductivity, flexibility and mechanical performance of RGO-CNF films above, it can be concluded that the film has potential application in the field of flexible electronic storage.

\subsection{Electrochemical performance}

Firstly, the CV (Fig. 8(a)) and GCD (Fig. 8(b)) characteristics of GO, GO-CNF, RGO-CNF30, RGO-CNF50, RGO-CNF70 samples are compared to reveal whether the hybrid film has the advantage in supercapacitor energy storage. In the Fig. 8(a), all curves show the shape of an approximate rectangle, implying good capacitance characteristics. However, compared with the CV curve of pure GO film, the CV curve of GOCNF film presents a much larger rectangular area, which demonstrates that a larger improvement of the specific capacitance is obtained due to the introduction of CNF spacer, the effective surface area of GO is greatly improved. Furthermore, when $\mathrm{GO}$ is reduced RGO, the CV curve of RGO-CNF film shows a more rectangular shape, and a larger specific surface area of the rectangle is obtained. These results are mainly due to the fact that the conductivity of RGO-CNF film is enhanced, and on the other hand, some wrinkles will be produced during the reduction of GO, which makes the effective specific surface area and layer spacing of RGO larger, being conducive to the storage and transmission of electrolyte ions. In addition, The proportion of GO and CNF has an important impact on the storage performance of RGOCNF film. Therefore, it is very important to investigate the different amount of CNF to optimize the storage performance of RGO-CNF film. The RGO-CNF30, RGO-CNF50 and RGO-CNF70 are selected to compare. Obviously, when the content of CNF increases from $30 \%$ to $50 \%$, the area of CV curve of RGO-CNF film increases correspondingly. However, when the content of CNF reaches $70 \%$, the area of $\mathrm{CV}$ curve becomes smaller. This is mainly due to the low content of CNF at the beginning, which is difficult to fully play its role as a spacer. However, if the content of CNF is too high, it will cause aggregation and loss of effective specific surface area. For the GCD curves of these samples (Fig. 8(b)), all curves show an approximately symmetrical triangle shape, and the discharge time of GO, GO-CNF and RGO-CNF films increases in turn, implying that the energy density increases in turn, which matches well with the CV results. Moreover, the RGO-CNF50 film shows the longest discharge time, which is also in line with the CV results. Therefore, the following electrochemical performance tests are based on RGO-CNF film. Fig. 8(c) gives a comparison of Nyquist plots of GO, GO-CNF50, RGO-CNF50 films. Apparently, in the low frequency region, shows a straight line closer to $90^{\circ}$, implying good capacitance characteristics. Furthermore, the relationship 
between the areal specific capacitance and stress of the RGO-CNF hybrid with CNF content is studied as shown in Fig. 8(d). It can be seen that with the increase of CNF content until $50 \%$, the areal specific capacitance of the RGO-CNF film shows an upward trend, reaching about $120 \mathrm{mF} \mathrm{cm}^{-2}$. At the same time, the tensile strength of the film also shows a rapid increase trend due to the addition of CNF. As the conclusion of CV and GCD, the specific capacitance of the RGO-CNF film decreases with the increase of CNF content, and the tensile strength increases slowly. In addition, to check the cycle stability, the CV curves of RGO-CNF hybrid under different potential window are compared (Fig. 8(e)). No matter which potential window, the CV curve of RGO-CNF film shows a similar trend, reflecting the better electrochemical stability of the RGO-CNF50 film. Furthermore, the cycle stability of RGO-CNF50 film is investigated as shown in Fig. 8(f). Apparently, after experiencing 5000 charge and discharge, the specific capacitance of RGO-CNT50 electrode did not decrease significantly, which indicated that the RGO-CNT50 electrode had good electrochemical cycle stability. Moreover, it can be seen from the inset of Fig. 8(f) that the GCD curve of RGO-CNF50 electrode material in the second cycle does not change significantly compared with that in $5000^{\text {th }}$, which also proves its good electrochemical cycle stability. In addition, the capacitance retention of RGO-CNF50 film is also studied under various current densities (Fig. 8(g)). It can be seen that the RGO-CNF50 film still maintains a higher capacitance retention under different current densities even in an ultra-high current density of $30 \mathrm{~mA} \mathrm{~cm}{ }^{-2}$, which demonstrates that an outstanding rate performance is obtained. More detailed information about the CV, GCD and EIS properties of each sample are displayed in Fig. S2 of the support information. According to the results of CV, GCD and EIS, the RGO-CNF50 electrode still has better electrochemical properties at different scan rates and current densities. Moreover, in the solid electrolyte, the CV test of the RGO-CNF50 electrode also shows a consistent trend in different potential windows (Fig. S3(a)), which indicates that the material has good stability in solid electrolyte. As we all known, the potential window of the solid electrolyte is wider than that of the aqueous electrolyte, so the energy density of the device can be further improved by using the solid electrolyte. Furthermore, comparison of electrochemical properties between RGO-CNF-PANI and RGO-CNF50 films in aqueous electrolyte (Fig. S3(b-d)), it can be seen that the RGO-CNF-PANI hybrid presents a great improved in electrochemical performance, which shows that the RGO-CNF film can be used as an effective substrate material to introduce other components to construct multi-stage composite materials, and further improve the energy storage characteristics of the materials.

\section{Conclusion}

A green CNF spacer has been successfully introduced into RGO interlayer by a simple liquid dispersion method, and further RGO-CNF films are successfully constructed by means of filtration and chemical reduction. The as-obtained RGO-CNF film by non carboniztion not only retains the good flexibility of natural CNF, but also as an effective spacer to increase the effective utilization specific surface area of RGO, and obtains excellent energy storage characteristics. In short, the film with good flexibility and mechanical strength shows a high specific capacitance of $120 \mathrm{mF} \mathrm{cm}{ }^{-2}\left(242 \mathrm{~F} \mathrm{~g}^{-1}\right)$, energy efficiency of $80 \%$, energy density of $536 \mu \mathrm{Wh} \mathrm{cm} \mathrm{cm}^{-2}\left(32 \mathrm{Wh} \mathrm{kg}^{-1}\right)$ and power density of $193 \mathrm{~mW} \mathrm{~cm}{ }^{-2}\left(53 \mathrm{~kW} \mathrm{~kg}^{-1}\right)$. The improved chemical properties are mainly attributed to the following three aspects: 1) CNF as a green 
spacer can effectively block the stacking of RGO, and obtain a large specific surface area to store more charges. 2) The cross-linked CNF forms more pore structures, which provide more space and channel for the storage and transport of electrolyte ions. 3) The properties of CNF give the composite film material good flexibility and mechanical strength. All these factors work together to endow the hybrid film with outstanding electrochemical storage performance and excellent mechanical properties. Last but not least, the CNF-RGO film shows a great potential in serving as a good substrate to load other substance to obtain energy storage materials with better comprehensive performance. Finally, it is hoped that our work can provide some references for the design and construction of green and sustainable high-performance GR-based composites.

\section{Declarations}

\section{Acknowledgments}

This work was supported by Supported by fund of National Natural Science Foundation of China (22078184); China Postdoctoral Science Foundation (2019M653853XB); Natural Science Foundation of Shaanxi Province (2020JQ-729); Opening Project of Guangxi Key Laboratory of Clean Pulp \& Papermaking and Pollution Control (2019KF21); Natural science advance research foundation of Shaanxi University of Science and Technology (2018QNBJ-03); National Demonstration Center for Experimental Light Chemistry Engineering Education (2018QGSJ02-13); High-level Foreign Experts Project (GDT20186100425), and Biomass Chemistry and Materials Acadamician Workstation Project in SUST (134090002).

\section{References}

Bi Z, Kong Q, Cao Y et al (2019) Biomass-derived porous carbon materials with different dimensions for supercapacitor electrodes: a review. Journal of Materials Chemistry A 7:16028-16045. https://doi.org/10.1039/C9TA04436A.

Chen D, Jiang K, Huang T et al (2020) Recent Advances in Fiber Supercapacitors: Materials, Device Configurations, and Applications. Advanced Materials 32:1901806.

https://doi.org/10.1002/adma.201901806.

Cheng Y, Zhang Y, Jiang $\mathrm{H}$ et al (2020) Coupled cobalt silicate nanobelt-on-nanobelt hierarchy structure with reduced graphene oxide for enhanced supercapacitive performance. Journal of Power Sources 448:227407. https://doi.org/10.1016/j.jpowsour.2019.227407.

Dang A, Li T, Xiong C et al (2018) Long-life electrochemical supercapacitor based on a novel hierarchically carbon foam templated carbon nanotube electrode. Composites Part B-Engineering 141:250-257. https://doi.org/10.1016/j.compositesb.2017.12.049. 
Deysher G, Shuck C, Hantanasirisakul K et al (2020) Synthesis of Mo4VAIC4 MAX Phase and TwoDimensional Mo4VC4 MXene with Five Atomic Layers of Transition Metals. ACS Nano 14:204-217. https://doi.org/10.1021/acsnano.9b07708

Du P, Dong Y, Kang H et al (2020) Superior cycle stability carbon layer encapsulated polyaniline nanowire core-shell nanoarray free-standing electrode for high performance flexible solid-state supercapacitors. Journal of Power Sources 449:227477. https://doi.org/10.1016/j.jpowsour.2019.227477.

El-Kady M, Shao Y, Kaner R et al (2016) Graphene for batteries, supercapacitors and beyond. Nature Review 1:1-14. https://doi.org/10.1038/natrevmats.2016.33

Fan L, Tu Q, Geng C et al (2020) High energy density and low self-discharge of a quasi-solid-state supercapacitor with carbon nanotubes incorporated redox-active ionic liquid-based gel polymer electrolyte. Electrochimica Acta 331:135425. https://doi.org/10.1016/j.electacta.2019.135425.

Li J, Liu Z, Zhang Q et al (2019) Anion and cation substitution in transition-metal oxides nanosheets for high-performance hybrid supercapacitors. Nano Energy 57:22-33.

https://doi.org/10.1016/j.nanoen.2018.12.011.

Li Z, Dall'Agnese Y, Guo J et al (2020) Flexible freestanding all-MXene hybrid films with enhanced capacitive performance for powering a flex sensor. Journal of Materials Chemistry A 8:16649-16660. https://doi.org/10.1039/D0TA05710J.

Liu Y, Wang W, Chen J et al (2020) Fabrication of porous lithium titanate self-supporting anode for high performance lithium-ion capacitor. Journal of Energy Chemistry 50:344-350.

https://doi.org/10.1016/j.jechem.2020.03.075

Marcano D, Kosynkin D, Berlin J et al (2010) Improved Synthesis of Graphene Oxide, ACS Nano 4:48064814. https://doi.org/10.1021/nn1006368

Nie G, Zhao X, Jiang J et al (2020) Flexible supercapacitor of high areal performance with vanadium/cobalt oxides on carbon nanofibers as a binder-free membrane electrode. Chemical Engineering Journal 402:126294. https://doi.org/10.1016/j.cej.2020.126294.

Peng Z, Zhong W (2020) Facile Preparation of an Excellent Mechanical Property Electroactive Biopolymer-Based Conductive Composite Film and Self-Enhancing Cellulose Hydrogel to Construct a High-Performance Wearable Supercapacitor. ACS Sustainable Chemistry \& Engineering 8:7879-7891. https://doi.org/10.1021/acssuschemeng.0c01118.

Sun Y, Wang Y, Liu L et al (2020) Towards the understanding of acetonitrile suppressing salt precipitation mechanism in a water-in-salt electrolyte for low-temperature supercapacitors. Journal of Materials Chemistry A 8:17998-18006. https://doi.org/17998-18006. 10.1039/D0TA04538A. 
Ten Elshof J, Yuan H, Rodriguez P (2016) Two-Dimensional Metal Oxide and Metal Hydroxide Nanosheets: Synthesis, Controlled Assembly and Applications in Energy Conversion and Storage. Advanced Energy Materials 6:1600355. https://doi.org/10.1002/aenm.201600355.

Thi Suong L, Thuy Kieu T, Van Ngoc H et al (2020) Synergetic design of enlarged surface area and pseudo-capacitance for fiber-shaped supercapacitor yarn. Nano Energy 67:104198.

https://doi.org/10.1016/j.nanoen.2019.104198.

Tong L, Gao M, Jiang C et al (2019) Ultra-high performance and flexible polypyrrole coated CNT paper electrodes for all-solid-state supercapacitors. Journal of Materials Chemistry A 7:10751-10760. https://doi.org/10.1039/C9TA01856E.

Wang Q, Zhang Y, Jiang H et al (2019) Designed mesoporous hollow sphere architecture metal (Mn, Co, $\mathrm{Ni})$ silicate: A potential electrode material for flexible all solid-state asymmetric supercapacitor. Chemical Engineering Journal 362:818-829. https://doi.org/10.1016/j.cej.2019.01.102.

Wei H, Wang H, Li A et al (2020) Advanced porous hierarchical activated carbon derived from agricultural wastes toward high performance supercapacitors. Journal of Alloys and Compounds 820:153111. https://doi.org/10.1016/j.jallcom.2019.153111.

Wu C, Unnikrishnan B, Chen I et al (2020) Excellent oxidation resistive MXene aqueous ink for microsupercapacitor application. Energy Storage Materials 25:563-571.

https://doi.org/10.1016/j.ensm.2019.09.026.

Wu T, Wu X, Li L et al (2020) Anisotropic Boron-Carbon Hetero-Nanosheets for Ultrahigh Energy Density Supercapacitors. Angewandte Chemie-International Edition 52:24008-24017. https://doi.org/10.1002/ange.202011523.

Xiao X, Zou L, Pang H et al (2020) Synthesis of micro/nanoscaled metal-organic frameworks and their direct electrochemical applications. Chemical Society Reviews 49:301-331. https://doi.org/10.1039/C7CS00614D.

Xiong C, Li B, Dang W et al (2020) Co/CoS nanofibers with flower-like structure immobilized in carbonated porous wood as bifunctional material for high-performance supercapacitors and catalysts. Materials \& Design 195:108942. https://doi.org/10.1016/j.matdes.2020.108942.

Xiong C, Li B, Liu H et al (2020) A smart porous wood-supported flower-like NiS/Ni conjunction with vitrimer co-effect as a multifunctional material with reshaping, shape-memory, and self-healing properties for applications in high-performance supercapacitors, catalysts, and sensors. Journal of Materials Chemistry A 8:10898-10908. https://doi.org/10.1039/D0TA03664A.

Xiong C, Li M, Nie S (2020) Non-carbonized porous lignin-free wood as an effective scaffold to fabricate lignin-free Wood@Polyaniline supercapacitor material for renewable energy storage application. Journal 
of Power Sources 471:228448. https://doi.org/10.1016/j.jpowsour.2020.228448.

Xiong C, Li M, Zhao W et al (2020) A smart paper@polyaniline nanofibers incorporated vitrimer bifunctional device with reshaping, shape-memory and self-healing properties applied in highperformance supercapacitors and sensors. Chemical Engineering Journal 396:125318. https://doi.org/10.1016/j.cej.2020.125318.

Xiong C, Li M, Zhao W et al (2020) Flexible N-Doped reduced graphene oxide/carbon Nanotube- $\mathrm{MnO}_{2}$ film as a Multifunctional Material for High-Performance supercapacitors, catalysts and sensors. Journal of Materiomics 6:523-531. https://doi.org/10.1016/j.jmat.2020.03.008.

Xiong C, Li T, Dang A et al (2016) Two-step approach of fabrication of three-dimensional $\mathrm{MnO}_{2}$-graphenecarbon nanotube hybrid as a binder-free supercapacitor electrode. Journal of Power Sources 306:602610. https://doi.org/10.1016/j.jpowsour.2015.12.056.

Xiong C, Li T, Zhao T et al (2016) Two-step approach of fabrication of three-dimensional reduced graphene oxide-carbon nanotubes-nickel foams hybrid as a binder-free supercapacitor electrode. Electrochimica Acta 217:9-15. https://doi.org/10.1016/j.electacta.2016.09.068.

Xiong C, Yang Q, Dang W et al (2020) Fabrication of eco-friendly carbon microtubes @ nitrogen-doped reduced graphene oxide hybrid as an excellent carbonaceous scaffold to load $\mathrm{MnO}_{2}$ nanowall (PANI nanorod) as bifunctional material for high-performance supercapacitor and oxygen reduction reaction catalyst. Journal of Power Sources 447:227387. https://doi.org/10.1016/j.jpowsour.2019.227387.

Xiong, C, Lin X, Liu H et al (2019) Fabrication of 3D Expanded Graphite-Based $\left(\mathrm{MnO}_{2}\right.$ Nanowalls and PANI Nanofibers) Hybrid as Bifunctional Material for High-Performance Supercapacitor and Sensor. Journal of the Electrochemical Society 166:A3965-A3971.

Zhang H, Tian Y, Wang S et al (2020) Highly stable flexible transparent electrode via rapid electrodeposition coating of Ag-Au alloy on copper nanowires for bifunctional electrochromic and supercapacitor device. Chemical Engineering Journal 399:125075.

https://doi.org/10.1016/j.cej.2020.125075.

Zhang M, Song Y, Yang D et al (2020) Redox Poly-Counterion Doped Conducting Polymers for Pseudocapacitive Energy Storage. Advanced Functional Materials 1:2006203.

https://doi.org/10.1002/adfm.202006203.

Zhao J, Jiang Y, Fan H et al (2017) Porous 3D Few-Layer Graphene-like Carbon for Ultrahigh-Power Supercapacitors with Well-Defined Structure-Performance Relationship. Advanced Materials 29:1604569. https://doi.org/10.1002/adma.201604569

Zhu L, Hao C, Wang X, Guo Y (2020) Fluffy Cotton-Like GO/Zn-Co-Ni Layered Double Hydroxides Form from a Sacrificed Template GO/ZIF-8 for High Performance Asymmetric Supercapacitors. ACS 


\section{Figures}

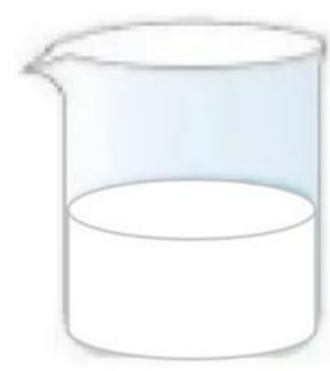

CNF
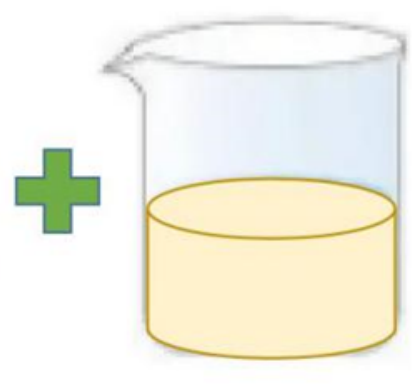

GO

\section{Ultrasonic}

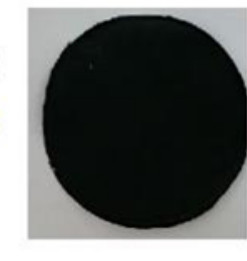

GO-CNF

\section{Hydrogen iodide}

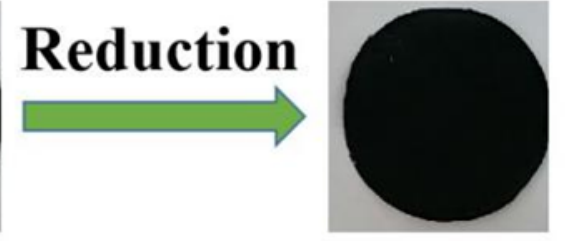

RGO-CNF

\section{Figure 1}

The schematic diagram of preparation process of RGO-CNF film. 


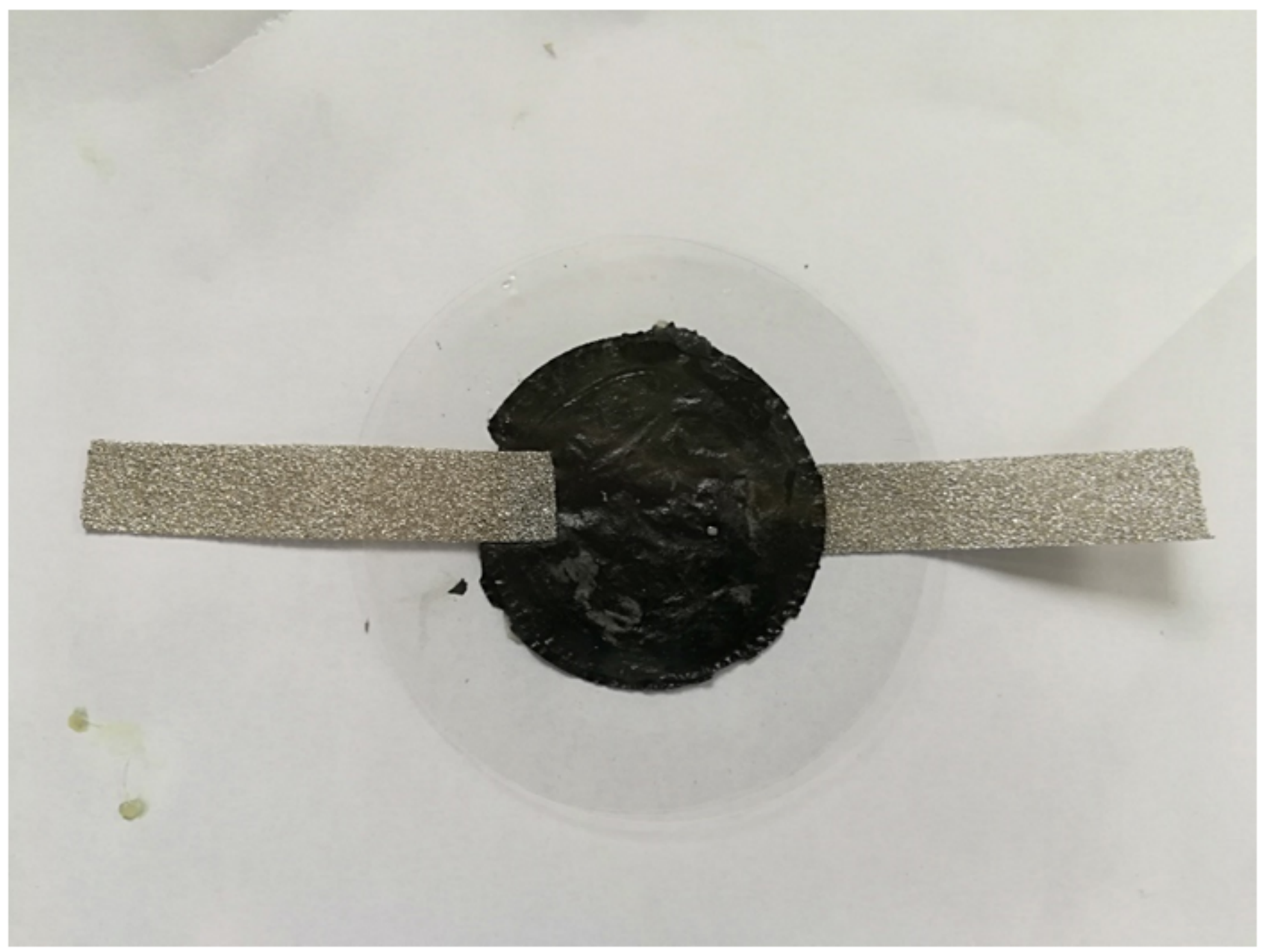

Figure 2

The schematic diagram of RGO-CNF supercapacitor device. 

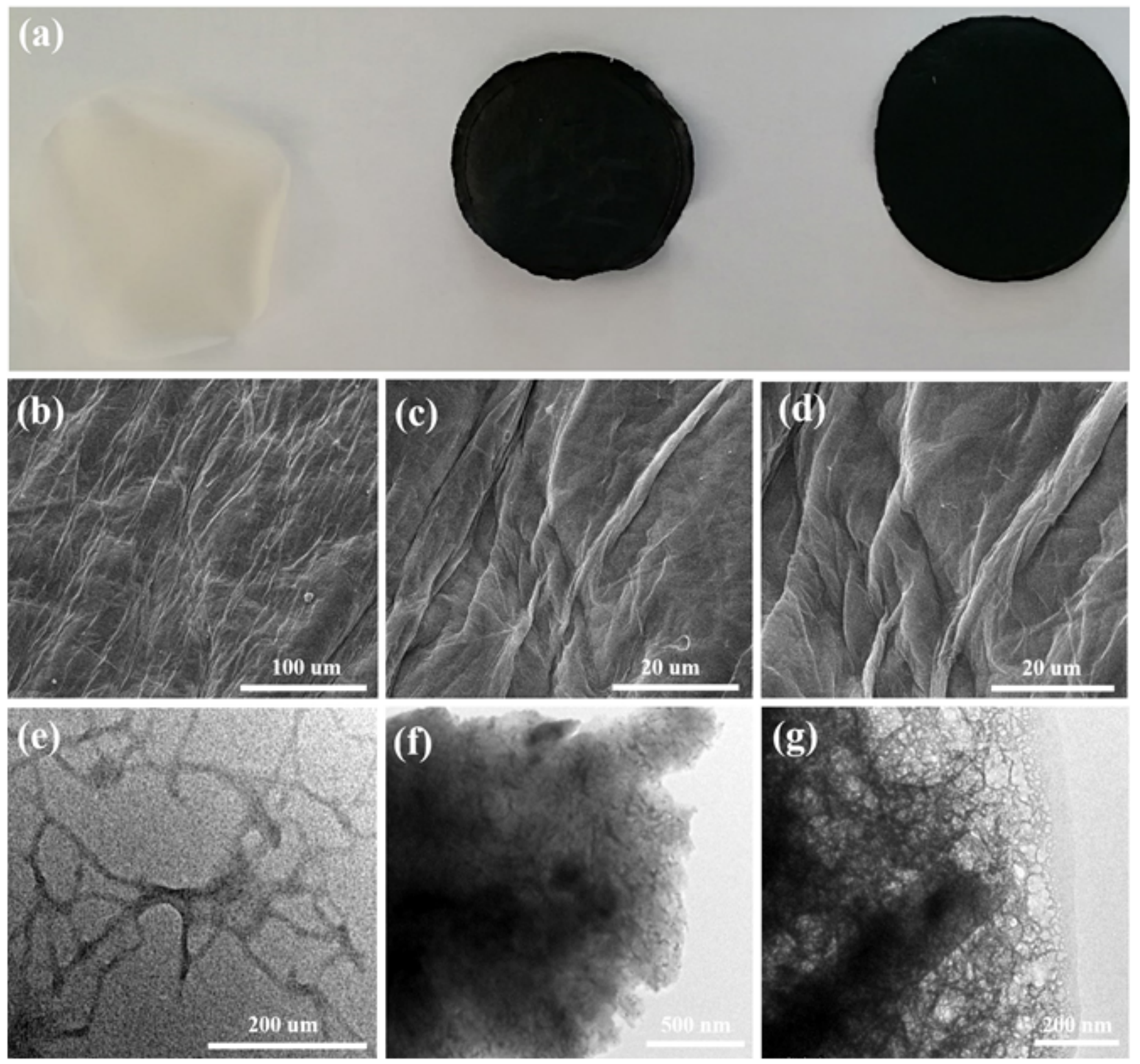

\section{Figure 3}

The digital graph of (a) pure CNF film, GO-CNF film and RGO-CNF film (from left to right). The SEM images of (b) pure CNF film, (c) GO-CNF film and (d) RGO-CNF film. The TEM images of (e) pure CNF film, (f) GO-CNF film and (g) RGO-CNF film. 

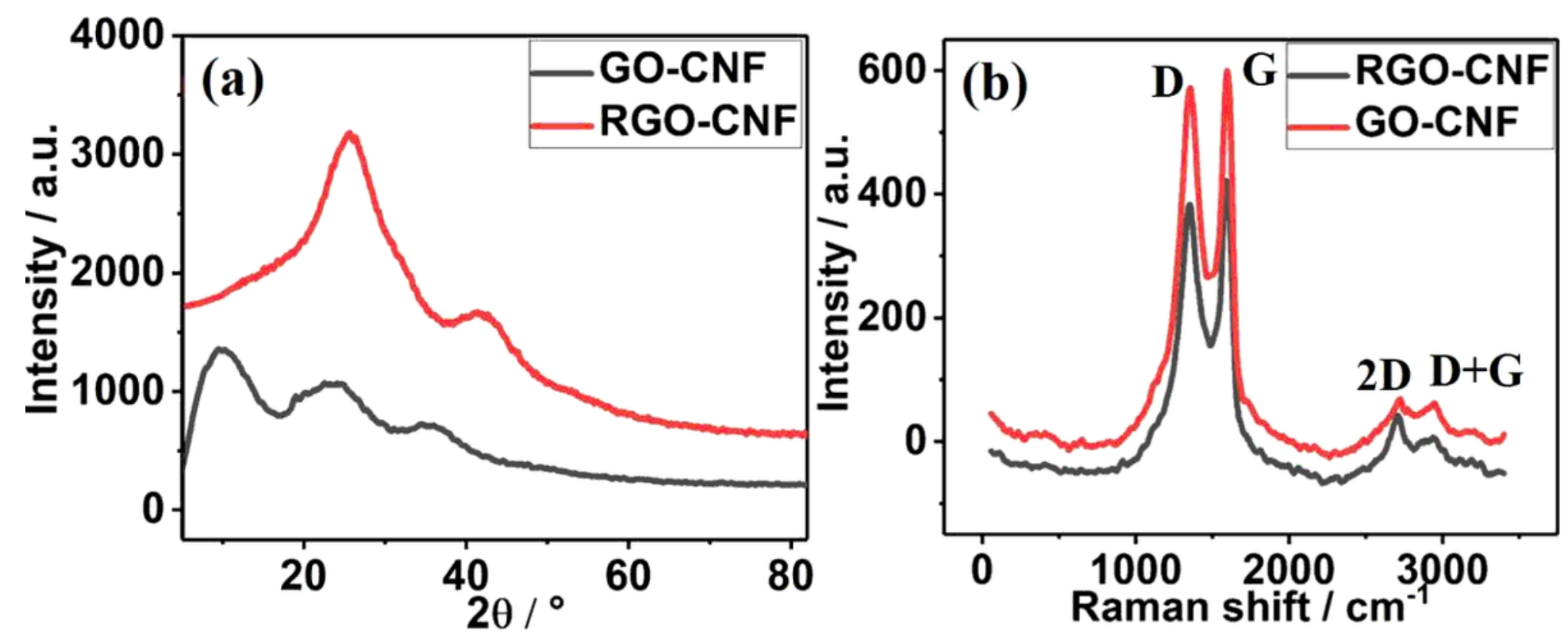

Figure 4

The (a) XRD and (b) Raman test of GO-CNF and RGO-CNF films.

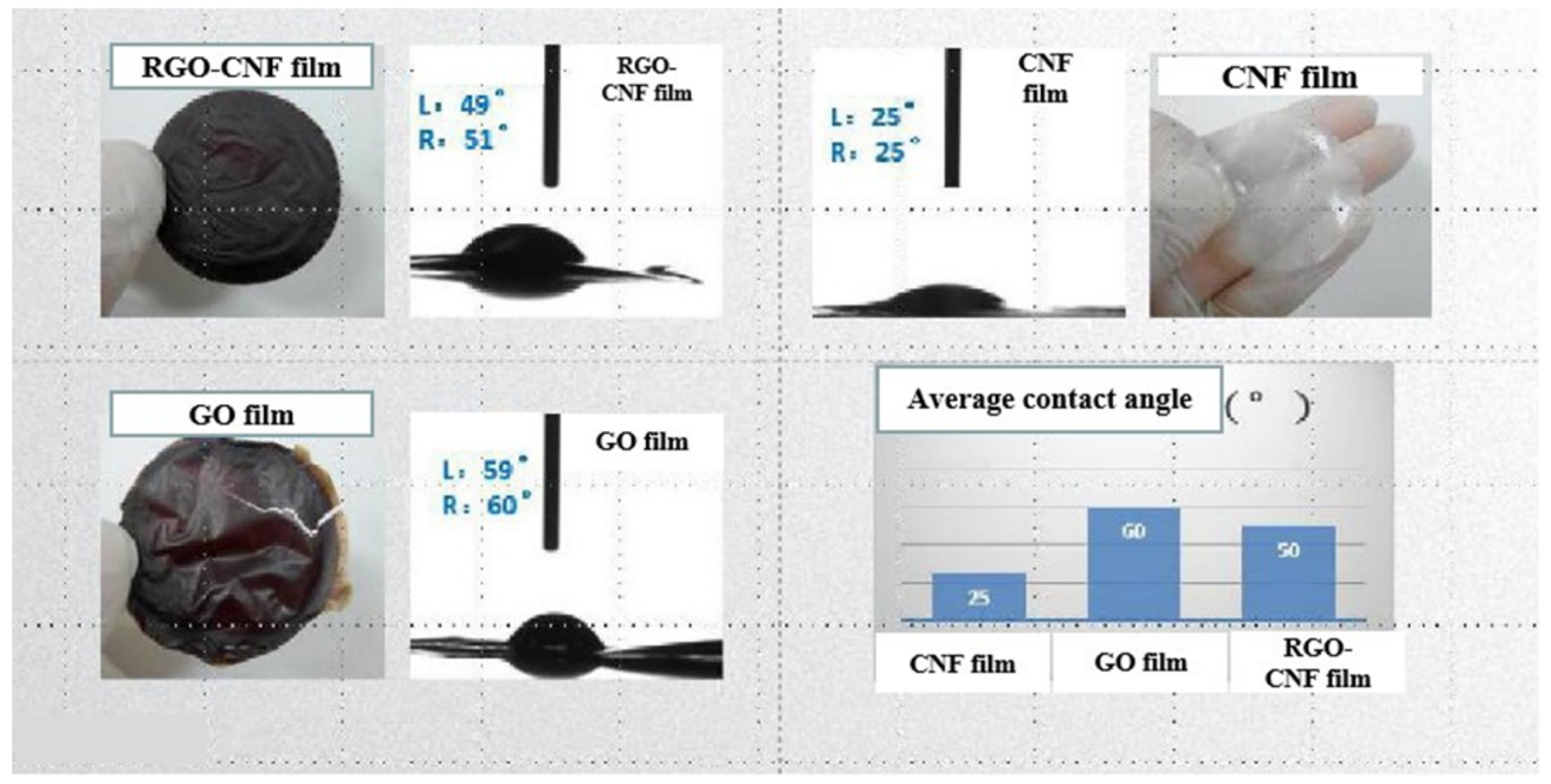

Figure 5

The hydrophilicity test of CNF, GO and RGO-CNF films. 

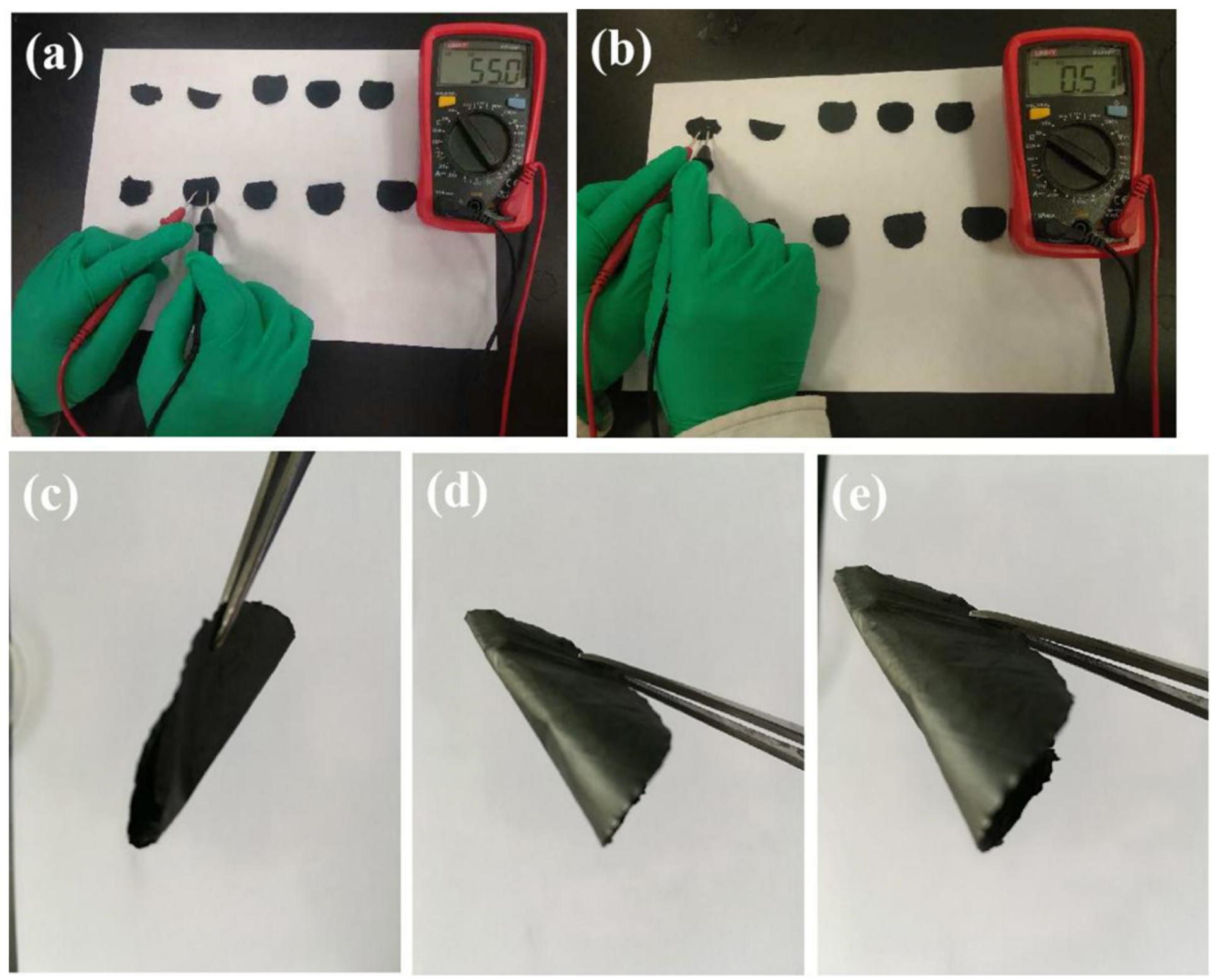

Figure 6

Measuring the resistance of (a) GO-CNF and (b) RGO-CNF films. (c-d) Digital maps showing flexibility of RGO-CNF films. 


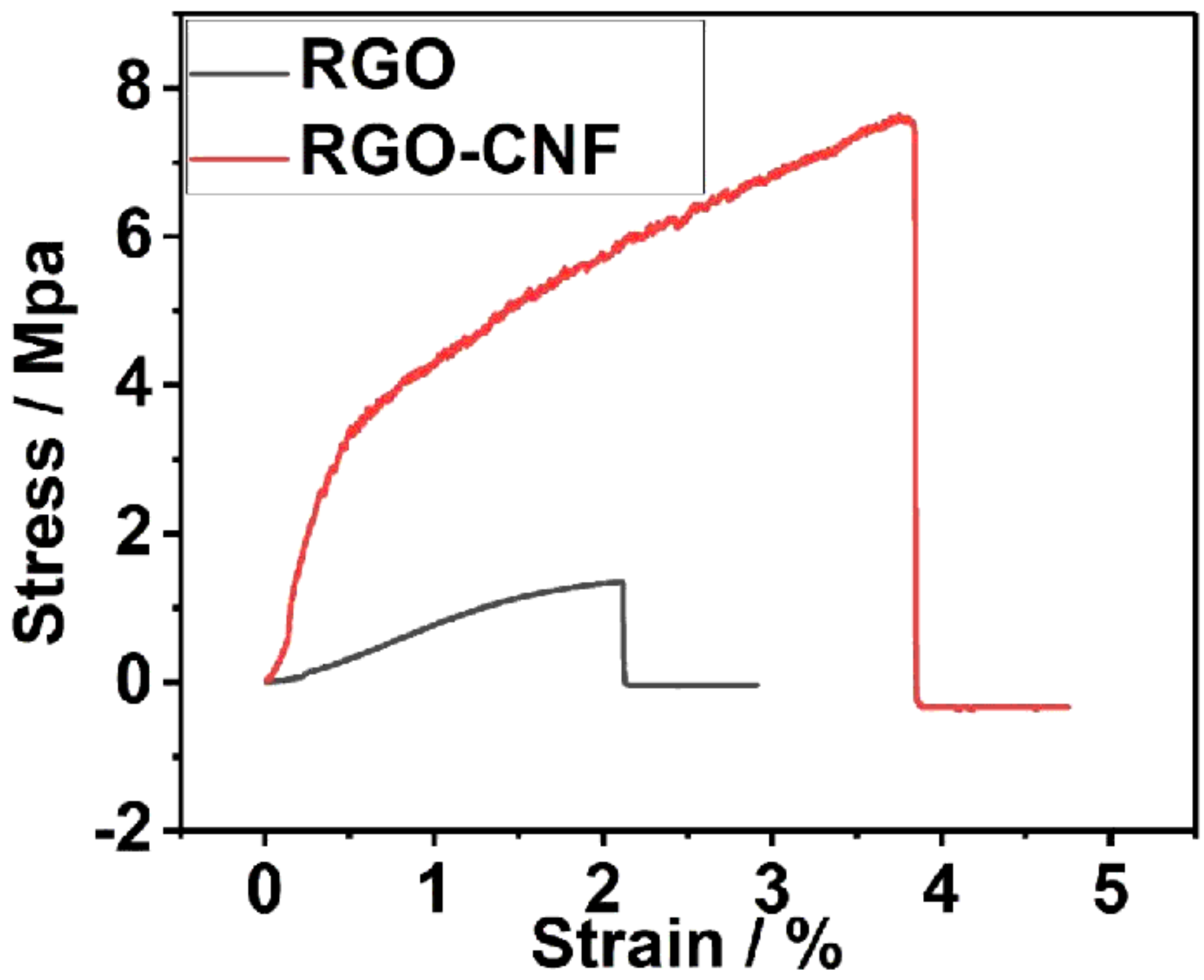

Figure 7

Comparison of tensile properties of RGO and RGO-CNF films. 

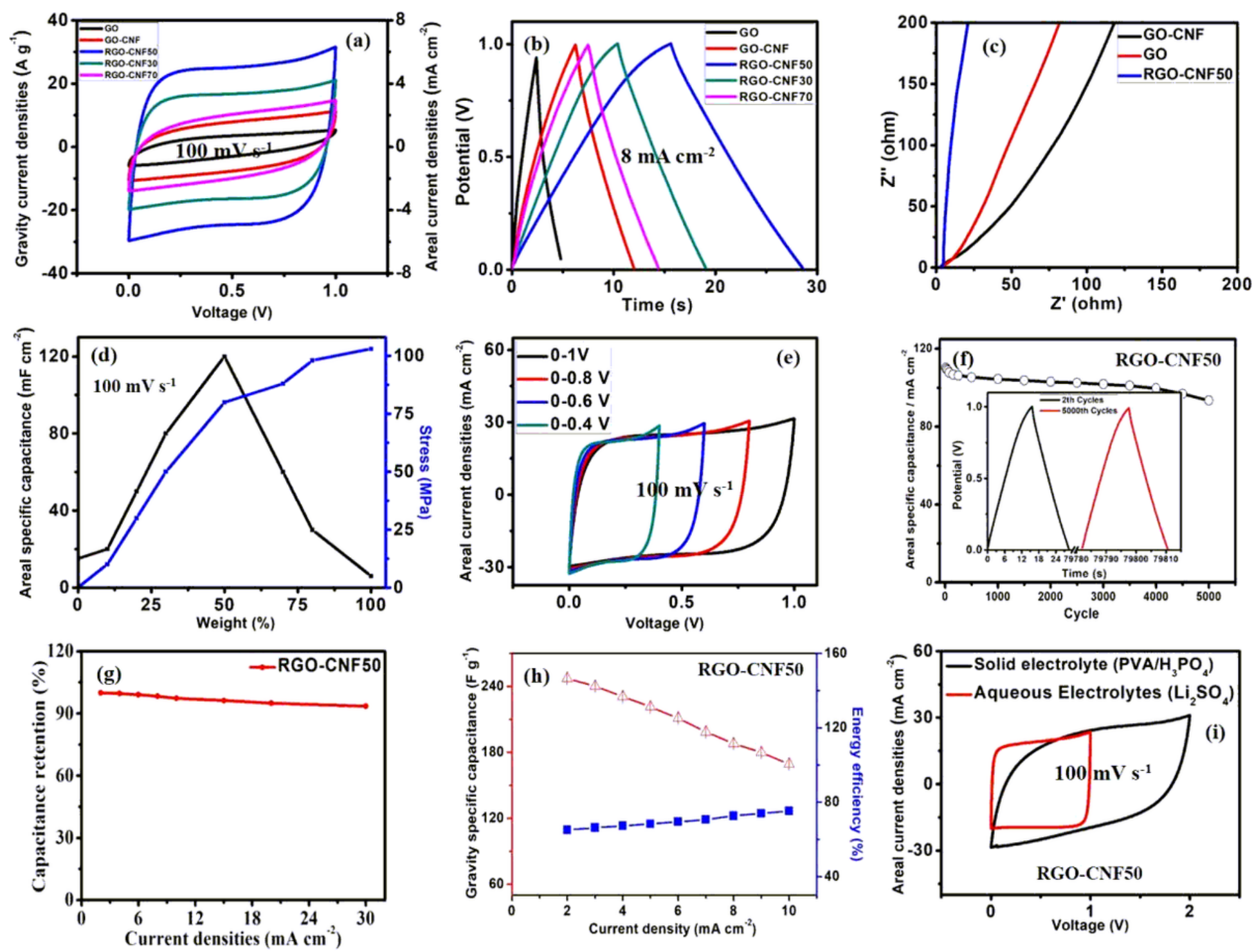

Figure 8

Comparison of (a) CV curves and (b) GCD curves of GO, GO-CNF, RGO-CNF30, RGO-CNF50, RGO-CNF70 samples, respectively. (c) Nyquist plots of GO, GO-CNF, RGO-CNF50 in the frequency range of 100000 to $0.01 \mathrm{~Hz}$. (d) The relationship between the areal specific capacitance and stress of the RGO-CNF hybrid with CNF content. (e) CV curves of RGO-CNF hybrid under different potential window. (f) The change of specific capacitance of RGO-CNF hybrid after experiencing 5000 cycles. (g) The capacitance retention of RGO-CNF hybrid under various current densities. (h) The change of specific capacitance and coulomb efficiency of RGO-CNF hybrid under different current densities. (i) Comparison of CV curves of RGO-CNF hybrid under aqueous and solid electrolytes.

\section{Supplementary Files}

This is a list of supplementary files associated with this preprint. Click to download.

- SupportInformation.doc 